\title{
Avaliação de Carcaças de Suínos da Raça Large White Utilizando Medidas Convencionais ${ }^{1}$
}

\author{
Rilke Tadeu Fonseca de Freitas ${ }^{2}$, Tarcísio de Moraes Gonçalves ${ }^{3}$, Antonio Ilson Gomes de \\ Oliveira $^{4}$, Daniel Furtado Ferreira ${ }^{5}$
}

\begin{abstract}
RESUMO - Visando estabelecer as medidas que melhor definem a quantidade de tecidos da carcaça de suínos, foram utilizados dados de rendimento de carne (RCARNE), rendimento de gordura (RGORD), rendimento de carcaça (RC), comprimento de carcaça pelo Método Brasileiro de Classificação de Carcaça (CCMB), comprimento de carcaça pelo Método Americano de Classificação de Carcaça (CCMA), espessura média de toucinho (ETM), espessura de toucinho a $4 \mathrm{~cm}$ da linha dorsal $\left(\mathrm{P}_{1}\right)$, espessura de toucinho a 6,5 $\mathrm{cm}$ da linha dorsal $\left(\mathrm{P}_{2}\right)$, espessura de toucinho a $8 \mathrm{~cm}$ da linha dorsal $\left(\mathrm{P}_{3}\right)$, área de olho de lombo (AOL), relação carne:gordura (RCG) e rendimento de pernil (RP), obtidos em 664 suínos Large White. Inicialmente, procurando elucidar qual a importância e possíveis estruturas de relação existentes entre as variáveis, utilizou-se a análise multivariada de agrupamento (cluster analysis) e, em seguida, adotou-se o modelo de regressão linear múltipla, sendo as análises executadas por intermédio do SAS. Numa primeira fase, estimou-se o coeficiente de determinação parcial (Tipo II) de cada variável e, em uma segunda fase, adotou-se o procedimento de seleção de equações ajustadas denominado “Backward”. Com exceção do RC, todas as medidas de carcaça avaliadas apresentaram correlação significativa com RCARNE e RGORD. As medidas ETM, $\mathrm{P}_{1}$, $\mathrm{P}_{2}$, $\mathrm{P}_{3}$ e RCG foram inversamente correlacionadas com RCARNE e diretamente correlacionadas com RGORD. Observou-se o contrário para as medidas CCMB, CCMA, AOL e RP. Na avaliação da qualidade da carcaça, para rendimento de carne são suficientes apenas as medidas ETM, AOL, RCG e RP, enquanto, para rendimento de gordura, além destas énecessária a mensuração da $\mathrm{P}_{1}$. As medidas de área de olho de lombo e espessura de toucinho, conforme propostas pelo MBCC mostraram-se como sendo as que melhor avaliam a quantidade de carne e gordura na carcaça.
\end{abstract}

Palavra-chave: análise de agrupamento, correlação, rendimento de carne e gordura

\section{Evaluation of Large White Pig Carcass Using Conventional Measures}

ABSTRACT - To establish the measures that best define the quality of the carcass of swine, data of lean meat yield (MY), fat yield (FY), dressing percentage (DP), carcass length for the Brazilian Method of Carcass Classification (CLBM), carcass length for the American Method of Carcass Classification (CLAM), average backfat thickness (ABT), backfat thickness $4 \mathrm{~cm}$ from the dorsal mid-line ( $\mathrm{P}_{1}$ ), backfat thickness $6.5 \mathrm{~cm}$ from the dorsal mid-line $\left(\mathrm{P}_{2}\right)$, backfat thickness $8 \mathrm{~cm}$ from the dorsal mid-line $\left(\mathrm{P}_{3}\right)$, loin eye area (LEA), lean:fat ratio (LFR) and ham yield (HY) were used, obtained in 664 Large White swine. Initially, in order to make clear the importance and possible relations structures among variables, the cluster analysis was used. Afterwards, the multiple regression linear model was used, using the SAS package. Firstly, it was estimated the partial correlation (Type II) of each trait to evaluate the importance of the quality variables of the carcass. Secondly, a procedure of adjusting equation selection named Backward was used. It was verified that, except for DP, all the measures of carcass classification evaluated presented correlation with MY and FY. The measures ABT, $\mathrm{P}_{1}, \mathrm{P}_{2}, \mathrm{P}_{3}$ and LFT were inversely correlated with MY and directly correlated with FY. The opposite was observed for the measures CLBM, CLAM, LEA and HY. ABT, AOL, RCG and RP are just enough to evaluate the quality of the carcass in terms of lean meat yield, and, in terms of FY, besides these measured previous it's necessary the evaluation of $\mathrm{P}_{1}$. The measures of loin eye area and average backfat thickness, as proposed by MBCC, shown as being what best evaluates the amount of meat and fat in the carcass.

Key Words: correlation, cluster analysis, meat and fat yield

\section{Introdução}

Nos últimos anos, houve rápida modernização e profissionalização na cadeia de produção de suínos. Atualmente, a indústria oferece produtos naturais, cortes especiais, alimentos semipreparados e lançamento de novos produtos industrializados. Essa diversificação é implementada sem grandes aumentos nos custos do sistema industrial tradicional, desde que contenha adequado processo de tipificação de carcaças.

\footnotetext{
1 Parte da dissertação de mestrado do último autor apresentada ao Programa de Pós-Graduação em Zootecnia da UFLA. Projeto financiado parcialmente pela FAPEMIG e CNPq.

2 Professor Adjunto do DZO da UFLA - CP 37 - 37200-000 - Lavras, MG. E.mail: rilke@ufla.br

3 Professor Adjunto do DZO da UFLA - Doutorando UNESP Botucatu - Bolsista CNPq. E.mail: tarcisio@ufla.br

4 Professor Titular Aposentado da UFLA. E.mail: ailson@ufla.br

5 Professor Adjunto do DEX da UFLA. E.mail: danielff@ufla.br
} 
Dependendo do processo de tipificação, inclui-se como qualidade de carcaça o rendimento ou a quantidade de carne na carcaça, a conformação visual, as medidas de tamanho da carcaça e a qualidade da carne. A tipificação de carcaças foi instituída oficialmente no Brasil pelo Ministério da Agricultura em 1981, sendo a Cooperativa Central Oeste Catarinense Ltda. a primeira empresa a adotá-la (Fávero, 1989).

No Brasil, mais precisamente nas indústrias do centro-sul, que têm adotado a classificação de carcaças como meio de bonificação para os produtores, as técnicas mais utilizadas consistem basicamente na medida, por meio de aparelhos ópticos (Irgang et al., 1998), da profundidade de gordura e músculo em determinados pontos da carcaça, durante o processo de abate, porém muitos fatores pré e pós-abate podem interferir nessa avaliação. Os aparelhos ópticos têm apresentado acurácia inferior aos aparelhos de ultrasonografia, principalmente no tocante à quantidade de músculo (Joyal et al., 1987).

A avaliação de carcaças de suínos pela ultrasonografia em tempo real tem sido adotada na maioria dos trabalhos de Avaliação e Classificação de Carcaças com ultra-som, realizados nos países desenvolvidos, graças à sua grande acurácia, variando 85 a $97 \%$ de correlação com as medidas tomadas diretamente nas carcaças, dependendo da característica avaliada, e também com as técnicas de abate utilizadas (Schinckel et al., 1994). Estas técnicas são muito caras e, por conseguinte, são viáveis apenas nos frigoríficos de médio e grande portes. Portanto, em alguns casos, as carcaças ainda são avaliadas utilizando-se o Método Brasileiro de Classificação de Carcaças (MBCC), complementado por outras medidas também feitas na carcaça. Apesar de não exigir equipamentos sofisticados e caros, este método requer a realização de várias medidas na carcaça, tornando-o trabalhoso e demorado. Assim, uma das maneiras de minimizar este problema é reduzir o número de medidas, eliminando as que menos contribuem para a avaliação da qualidade das carcaças.

Considerando-se esses aspectos, objetivou-se estabelecer as medidas de classificação de carcaça, realizadas com equipamento ou aparelhos de medição convencionais, suficientes para avaliar a qualidade da carcaça de suínos.

\section{Material e Métodos}

O experimento foi conduzido no período de maio de 1997 a março de 2000, no setor de suinocultura do Departamento de Zootecnia da Universidade Federal de Lavras (UFLA). Os 664 animais da raça Large White (machos castrados e fêmeas) foram criados em regime intensivo com ração e água à vontade. As rações foram formuladas para satisfazer às exigências nutricionais dos animais, segundo as recomendações de Rostagno et al. (1994).

Quando os animais apresentaram aproximadamente $95 \mathrm{~kg}$ de peso vivo, foram submetidos ao processo de abate, iniciado com jejum alimentar (24 horas) e líquido (12 horas). Este processo envolveu: pesagem após o jejum, atordoamento, sangria, higienização, evisceração, toalete, divisão e pesagem da carcaça, identificação e resfriamento em câmara fria com temperatura de 2 a $4^{\circ} \mathrm{C}$ por 24 horas, como preconizado pela Associação Brasileira de Criadores de Suínos, (ABCS, 1973). Por convenção, a cauda permaneceu na meia-carcaça esquerda.

Depois de resfriadas, as meia-carcaças esquerdas forampesadas e classificadas segundo o Método Brasileiro de Classificação de Carcaças (MBCC), descrito pela ABCS (1973), e dissecadas de acordo com a técnica de Cuthbertson(1968), modificada por Almeida Neto(1992). A carcaça foi dividida em: paleta, costado, barriga e pernil, separando-se carne, gordura (subcutânea e intramuscular) e pele juntas, ossos e resíduo.

Rendimento de carcaça, comprimento de carcaça pelos métodos brasileiro e americano, espessura de toucinho média, $\mathrm{P}_{1}, \mathrm{P}_{2}, \mathrm{P}_{3}$, área de olho de lombo, relação carne:gordura, rendimento de pernil e porcentagem de carne e de gordura foram analisados.

Para estudar as medidas que representam a qualidade da carcaça de suínos e suas correlações, inicialmente adotou-se o seguinte modelo de regressão linearmúltipla:

$$
Y_{i}=b_{0}+b_{1} X_{1 i}+b_{2} X_{2 i}+b_{3} X_{3 i}+\ldots .+b_{10 i} X_{10 i}+\varepsilon_{i}
$$

em que $\mathrm{Y}_{\mathrm{i}}$ representa as variáveis que determinam diretamente a qualidade da carcaça (RCARNE e RGORD) na i-ésima parcela; $\mathrm{X}_{1 \mathrm{i}}, \ldots, \mathrm{X}_{10 \mathrm{i}}$, representam o RC, CCMB, CCMA, ETM, $\mathrm{P}_{1}, \mathrm{P}_{2}, \mathrm{P}_{3}$, AOL, RCG e $\mathrm{RP}$ da i-ésima parcela, respectivamente, que foram descritos anteriormente; $\mathrm{e}_{\mathrm{i}}$ representa o desvio de regressão da i-ésima observação assumido $\operatorname{NID}\left(0, \sigma_{\mathrm{e}}{ }^{2}\right)$. 
Para elucidar a estrutura de relações entre as variáveis independentes do referido modelo, inicialmente estimaram-se as correlações de Pearson (Draper \& Smith, 1981) entre todos os pares possíveis, utilizando-se o Proc Reg do SAS (1995), e, posteriormente, a análise multivariada (cluster analysis), conforme Johnson \& Wichern (1988). Adotou-se o método do vizinho mais distante (complete linkage) e obteve-se o dendograma por meio do programa Statistica.

Finalmente, ajustou-se o modelo de regressão apresentado anteriormente. Na primeira fase, estimou-se o coeficiente de determinação parcial de cada variável (Tipo II), no modelo completo e, na segunda fase, empregou-se o procedimento de seleção de equações ajustadas denominado Backward (Draper \& Smith, 1981). O procedimento, realizado passo a passo, eliminou as variáveis de menor importância em cada etapa. A variável com menor valor de F parcial, cujo teste de hipótese $\mathrm{H}_{0}$ : $\mathrm{b}_{\mathrm{i}}=0(\mathrm{i}=1,2, \ldots, 10)$ não foi rejeitado (F não-significativo a 1\%), foi elimi- nada naquele passo, repetindo-se até que houvesse apenas variáveis com $\mathrm{F}$ parcial significativas no modelo. As variáveis remanescentes foram classificadas quanto à importância, de acordo com os maiores valores de $\mathrm{F}$ parcial (ou dos valores em módulo do $\mathrm{t}$ de Student) e as eliminadas foram classificadas pela sua importância, de acordo com a ordem de eliminação no processo de Backward.

\section{Resultados e Discussão}

As estatísticas descritivas (Tabela 1) comprovaram que o rendimento de carne (RCARNE) na carcaça foi de $49,49 \%(0,12 \%)$ com um CV de $6,28 \%$. A porcentagem de rendimento de gordura (RGORD) média foi de 27,38 $(0,13)$ com um CV de 12,56\%. A grande amplitude dos dados de RCARNE e RGORD, que repercutiu nos valores dos coeficientes de variação, se deve à heterogeneidade do plantel utilizado, constituído de animais não-selecionados.

Tabela 1 - Média, desvio-padrão, erro-padrão, mínimo, máximo e coeficiente de variação das variáveis avaliadas em 668 animais

Table 1 - Means, standard deviation, standard error, minimum, maximum and coefficient of variation of traits of the Large White swine carcass

\begin{tabular}{|c|c|c|c|c|c|c|}
\hline $\begin{array}{l}\text { Variável }^{1} \\
\text { Trait }^{1}\end{array}$ & $\begin{array}{l}\text { Média } \\
\text { Mean }\end{array}$ & $\begin{array}{c}\text { Desvio-padrão } \\
\text { Standard deviation }\end{array}$ & $\begin{array}{l}\text { Erro-padrão } \\
\text { Standard error }\end{array}$ & $\begin{array}{l}\text { Mínimo } \\
\text { Minimum }\end{array}$ & $\begin{array}{l}\text { Máximo } \\
\text { Maximum }\end{array}$ & $\mathrm{CV}(\%)^{2}$ \\
\hline $\begin{array}{l}\text { RCARNE } \\
L M Y(\%)\end{array}$ & 49,49 & 3,11 & 0,12 & 39,26 & 57,20 & 6,28 \\
\hline $\begin{array}{l}\text { RGORD } \\
F Y(\%)\end{array}$ & 27,38 & 3,44 & 0,13 & 17,70 & 38,31 & 12,56 \\
\hline $\begin{array}{l}\mathrm{RC} \\
D P(\%)\end{array}$ & 81,31 & 2,71 & 0,11 & 71,28 & 91,78 & 3,34 \\
\hline $\begin{array}{l}\text { CCMB } \\
C L B M(\mathrm{~cm})\end{array}$ & 93,23 & 3,12 & 0,12 & 83,00 & 102,00 & 3,35 \\
\hline $\begin{array}{l}\text { CCMA } \\
C L A M(\mathrm{~cm})\end{array}$ & 78,34 & 2,90 & 0,11 & 70,00 & 95,00 & 3,70 \\
\hline $\begin{array}{l}\mathrm{ETM}(\mathrm{mm}) \\
A B T\end{array}$ & 35,39 & 5,06 & 0,20 & 17,83 & 50,67 & 14,28 \\
\hline $\begin{array}{l}\mathrm{P}_{1}(\mathrm{~mm}) \\
\mathrm{P}_{2}(\mathrm{~mm}) \\
\mathrm{P}_{3}(\mathrm{~mm}) \\
\mathrm{AOL}\left(\mathrm{cm}^{2}\right)\end{array}$ & $\begin{array}{l}22,84 \\
24,94 \\
27,27 \\
31,99\end{array}$ & $\begin{array}{l}5,42 \\
6,96 \\
7,53 \\
4,57\end{array}$ & $\begin{array}{l}0,21 \\
0,27 \\
0,29 \\
0,18\end{array}$ & $\begin{array}{r}10,00 \\
8,00 \\
9,00 \\
19,00\end{array}$ & $\begin{array}{l}45,00 \\
44,00 \\
49,00 \\
50,00\end{array}$ & $\begin{array}{l}23,72 \\
27,93 \\
27,60 \\
14,29\end{array}$ \\
\hline $\begin{array}{l}\text { LEA } \\
\text { RCG }\end{array}$ & 0,78 & 0,19 & 0,01 & 0,26 & 1,37 & 23,88 \\
\hline $\begin{array}{l}M F R \\
\mathrm{RP}(\%) \\
H Y\end{array}$ & 30,59 & 1,78 & 0,07 & 23,98 & 34,80 & 5,83 \\
\hline
\end{tabular}

${ }^{1}$ RCARNE: rendimento de carne, RGORD: rendimento de gordura e pele, RC: rendimento de carcaça, CCMB: comprimento de carcaça pelo método brasileiro, CCMA: comprimento de carcaça pelo método americano, ETM: espessura média de toucinho, $\mathrm{P}_{1}$ : espessura de toucinho a $4 \mathrm{~cm}$ da linha dorsal, $\mathrm{P}_{2}$ : espessura de toucinho a $6,5 \mathrm{~cm}$ da linha dorsal, $\mathrm{P}_{3}$ : espessura de toucinho a $8 \mathrm{~cm}$ da linha dorsal, AOL: área de olho de lombo, RCG: relação carne:gordura pelo MBCC, RP: rendimento de pernil.

2 Coeficiente de variação (Coefficient of variation).

1 LMY: lean meat yield; FY: fat yield; DP: dressing percentage; CLBM: carcass length by Brazilian method; CLAM: carcass length by American method; ABT: average backfat thickness; $P_{1}$ : backfat thickness $4 \mathrm{~cm}$ from the dorsal mid-line: $P_{2}$ : backfat thickness $6.5 \mathrm{~cm}$ from the dorsal mid-line: $P_{3}$ : backfat thickness $8 \mathrm{~cm}$ from the dorsal mid-line: LEA: Loin eye area; MFR: lean:fat ratio; HY: ham yield.

R. Bras. Zootec., v.33, n.6, p.2037-2043, 2004 (Supl. 2) 


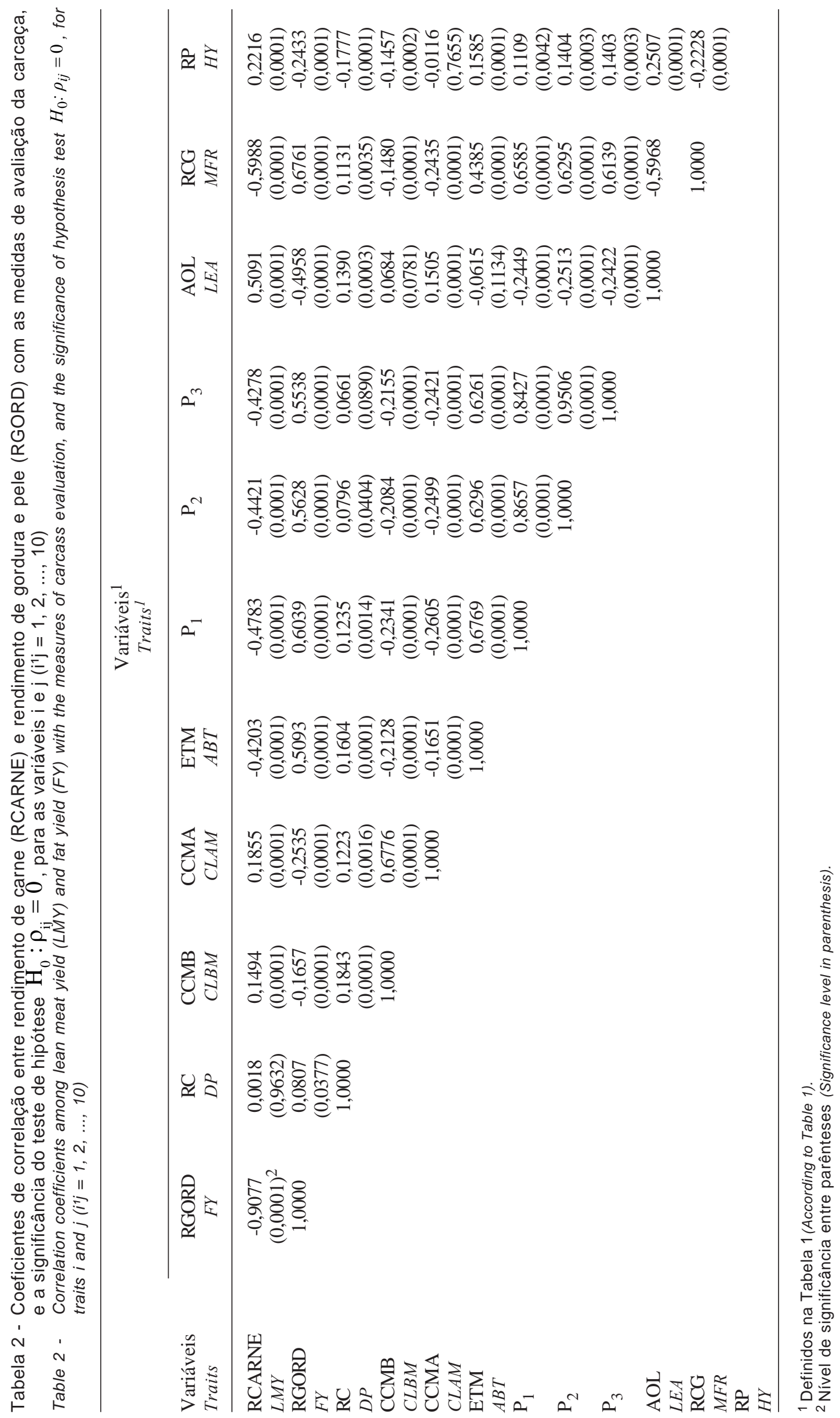


O rendimento de carcaça (RC) obtido (81,31\%,) está dentro dos limites reportados na literatura, entretanto observou-se elevada espessura de toucinho $\operatorname{ETM}(35,39 \mathrm{~mm})$, maior que as encontradas por Catalan (1986), de 21,3 mm, e Almeida Neto (1992), de 30,7 mm. Semelhantemente, os valores de espessuras de toucinho a $4 \mathrm{~cm}$ da linha dorsal $\left(\mathrm{P}_{2}\right)$ também se apresentaram superiores às médias encontradas por Oliveira (1988). Esses resultados indicam que os animais avaliados apresentaram alta porcentagem de gordura na carcaça, o que pode ser explicado pela reduzida seleção à qual esse plantel foi submetido.

As demais estatísticas descritivas das características de classificação de carcaça apresentaram valores semelhantes aos encontrados na literatura. Os resultados de RCARNE e de RGORD foram coerentes com aqueles descritos por Quijandria et al. (1970), Oliveira (1988) e Almeida Neto (1992).

Pelos coeficientes de correlação entre as variáveis estudadas (Tabela 2), verificou-se que as medidas ETM, espessura de toucinho a $4 \mathrm{~cm}$ da linha dorsal $\left(\mathrm{P}_{1}\right), \mathrm{P}_{2}$, espessura de toucinho a 8 cm da linha dorsal $\left(\mathrm{P}_{3}\right)$ e a relação carne:gordura
(RCG) tiveram correlações negativas com RCARNE, quando estas foram avaliadas individualmente, as quais, embora não muito altas, foram significativas $(\mathrm{P}<0,01)$.

O fato de as correlações serem negativas é facilmente explicável, posto que estas medidas estão associadas à quantidade de gordura, que é inversamente proporcional à quantidade de carne. As variáveis CCMB, CCMA, AOL e RP apresentaram correlação positiva $(\mathrm{P}<0,01)$ com RCARNE, como relatado por Almeida Neto (1992), que encontrou, para RP e AOL, correlação genética positiva com algumas características que avaliam a quantidade de carne na carcaça. A única medida não correlacionada com RCARNE foi RC, contrariando os resultados observados por Schneider (1982), Oliveira (1988) e Almeida Neto (1992), que obtiveram relação inversa entre RC e RCARNE.

A variável que apresentou maior correlação positiva estimada com RCARNE foi AOL, sendo que a variável com a maior correlação negativa foi RCG. Estes resultados indicam que a AOL é um indicador da quantidade de carne presente no animal, o que
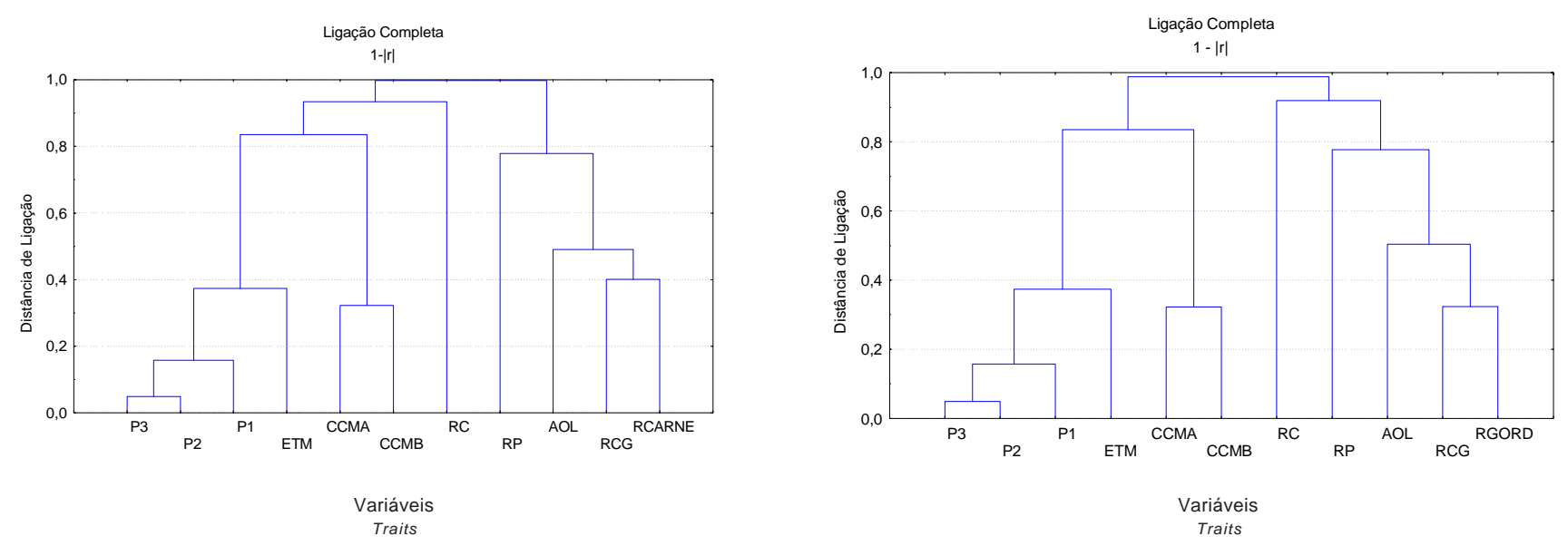

Figura 1 - Dendograma resultante da análise de agrupamento, utilizando como medida de dissimilaridade 1-| $r$ | ( $r$ : correlação de Pearson) e o método do vizinho mais distante, para a variável rendimento de carne (RCARNE) - A - e rendimento de gordura e pele (RGORD) - B - e as medidas rendimento de carcaça (RC), comprimento de carcaça pelo método brasileiro (CCMB), comprimento de carcaça pelo método americano (CCMA), espessura média de toucinho $(E T M)$, espessura de toucinho a $4 \mathrm{~cm}$ da linha dorsal $\left(P_{1}\right)$, espessura de toucinho a $6,5 \mathrm{~cm}$ da linha dorsal $\left(\mathrm{P}_{2}\right)$, espessura de toucinho a $8 \mathrm{~cm}$ da linha dorsal $\left(\mathrm{P}_{3}\right)$, área de olho de lombo (AOL), relação carne:gordura pelo MBCC (RCG) e rendimento de pernil (RP).

Figure 1 - Tree diagram built from cluster analysis, using as dissimilarity measure 1-| $r \mid$ ( $r$ : Pearson correlation) and the method of complete linkage for the trait lean yield (RCARNE) - $A$ - and fat yield (RGORD) - $B$ - and the measures dressing percentage $(R C)$, carcass length by Brazilian method (CCMB), carcass length by American method (CCMA), average backfat thickness $(E T M)$, backfat thickness $4 \mathrm{~cm}$ from the dorsal mid-line $\left(P_{1}\right)$, backfat thickness $6.5 \mathrm{~cm}$ from the dorsal mid-line $\left(P_{2}\right)$, backfat thickness $8 \mathrm{~cm}$ from the dorsal mid-line $\left(P_{3}\right)$, Loin eye area $(A O L)$, lean:fat ratio $(R C G)$, ham yield $(R P)$.

R. Bras. Zootec., v.33, n.6, p.2037-2043, 2004 (Supl. 2) 
coincide com os resultados observados por Smith \& Pearson (1986) e McLaren et al. (1987), enquanto uma seleção para redução na RCG também poderá aumentar RCARNE.

Com relação aos coeficientes estimados para RGORD, nota-se (Tabela 2) que CCMB, CCMA, AOL e RP apresentaram correlações negativas $(\mathrm{P}<0,01)$. Este resultado é considerado normal tendo em vista que estas variáveis estão diretamente associadas com RCARNE. As variáveis ETM, $\mathrm{P}_{1}$, $\mathrm{P}_{2}, \mathrm{P}_{3}$ e RCG apresentaram, como já esperado, correlações positivas $(\mathrm{P}<0,01)$ com RGORD, enquanto RC apresentou baixa correlação positiva, diferindo do resultado encontrado por Almeida Neto (1992), que observou correlação genética positiva mais elevada. As espessuras medidas na altura da última costela tiveram correlações semelhantes com RGORD, sendo iguais a 0,$60 ; 0,56$ e 0,55 , para $\mathrm{P}_{1}, \mathrm{P}_{2}$, $\mathrm{P}_{3}$, respectivamente. Este resultado contraria os encontrados na literatura, uma vez que Kempster et al. (1981) afirmam que P2 é a principal medida de gordura utilizada no processo de avaliação de carcaça na Comunidade Européia.

A análise de agrupamento forneceu os resultados apresentados nos dendogramas da Figura 1. Adotando-se o ponto de corte em torno de 0,45 na distância de ligação (Figura 1-A) e de 0,38 (Figura 1-B), de forma arbitrária como é realizado pela maioria da literatura especializada (Johnson \& Wichern, 1988), verificou-se que, na Figura 1-A, formaram-se seis grupos distintos de variáveis: RCARNE e RCG; AOL; RP; RC, CCMA e CCMB; e, finalmente, $\mathrm{P}_{1}, \mathrm{P}_{2}, \mathrm{P}_{3}$ e ETM. Os resultados obtidos para RCARNE permitem supor

Tabela 3 - Coeficiente de determinação parcial (tipo II) entre as variáveis consideradas no modelo com a variável que avalia o rendimento de carne (RCARNE) e significância do teste da hipótese $\mathrm{H}_{0}: \rho_{\mathrm{ij}}$ (parcial) $=0$

Table 3 - Partial correlation (type II) between the traits in model with the trait meat lean yield and the significance of the hypothesis test

\begin{tabular}{lcc}
\hline $\begin{array}{l}\text { Variável } \\
\text { Trait }\end{array}$ & $\begin{array}{c}\text { Coeficiente de determinação } \\
\text { parcial (\%) } \\
\text { Partial correlation }\end{array}$ & $\begin{array}{c}\text { Significância } \\
\text { Significance }\end{array}$ \\
\hline $\operatorname{ETM}(A B T)$ & 11,51 & $<0,0001$ \\
AOL $(L E A)$ & 9,55 & $<0,0001$ \\
RCG $(L F R)$ & 4,92 & $<0,0001$ \\
RP $(H Y)$ & 3,07 & $<0,0001$ \\
\hline
\end{tabular}

1 Definidos na Tabela 1 (According to Table 1).

R. Bras. Zootec., v.33, n.6, p.2037-2043, 2004 (Supl. 2) que há maior associação da variável RCG com o rendimento de carne (RCARNE), seguida pela AOL, sendo que as demais têm pouco relacionamento com RCARNE. Verificou-se também que as variáveis $\mathrm{P}_{3}$ e $\mathrm{P}_{2}$ são as mais similares, por possuírem o menor ponto de fusão (menor distância de ligação).

Consta, na Figura 1-B, o dendograma substituindo a variável RCARNE por RGORD. Verificou-se a mesma tendência para formação dos grupos, com sete grupos neste caso, sendo que a variável ETM formou um grupo independente. As espessuras de toucinho $\mathrm{P}_{1}, \mathrm{P}_{2}, \mathrm{P}_{3}$ apresentaram bastante similaridade, ou seja, devem conter grande parte de informações redundantes mutuamente.

Para identificar possíveis redundâncias entre as medidas estudadas para a explicação dos rendimentos de carne e de gordura (RCARNE e RGORD), estimaram-se os coeficientes de determinação parcial. Os valores da Tabela 3, referentes à RCARNE, apresentaram também a respectiva significância do teste da hipótese $\mathrm{H}_{0}: \mathrm{r}_{\mathrm{ij}}$ (parcial) = 0, com $\mathrm{i}=1($ RCARNE) e $\mathrm{j}=2,3, \ldots, 4$ (para as demais medidas). Para esta variável, as medidas RC, CCMA, CCMB, $\mathrm{P}_{1}, \mathrm{P}_{2}$ e $\mathrm{P}_{3}$ tiveram os menores coeficientes de determinação parcial, todos não-significativos ( $\mathrm{P}>0,01)$. RCG, AOL, ETM e RP, mesmo tendo explicação redundante com as demais, apresentaram maiores coeficientes de determinação parcial $(\mathrm{P}<0,01)$, sendo as medidas com as maiores estimativas as que mais influenciam RCARNE.

Este resultado está de acordo com os obtidos por Schneider et al. (1982), Oliveira (1988) e Almeida Neto (1992), que observaram que as variáveis ETM,

Tabela 4 - Coeficiente de determinação parcial (tipo II) entre as variáveis consideradas no modelo com a variável que avalia o rendimento de gordura (RGORD) e significância do teste da hipótese

Table 4 - Partial correlation (type II) between the traits in model with the trait fat yield and the significance of the hypothesis test

\begin{tabular}{lcc}
\hline $\begin{array}{l}\text { Variável }^{1} \\
\text { Trait }\end{array}$ & $\begin{array}{c}\text { Coeficiente de determinação } \\
\text { parcial (\%) } \\
\text { Partial correlation }\end{array}$ & $\begin{array}{c}\text { Significância } \\
\text { Significance }\end{array}$ \\
\hline ETM $(A B T)$ & 8,46 & $<0,0001$ \\
$\mathrm{P}_{1}$ & 5,48 & $<0,0001$ \\
AOL $(L E A)$ & 8,17 & $<0,0001$ \\
RCG $($ LFR $)$ & 3,03 & $<0,0001$ \\
RP $(H Y)$ & 8,12 & $<0,0001$ \\
\hline
\end{tabular}

${ }^{1}$ Definidos na Tabela 1 (According to Table 1). 
AOL, RCG e RP são boas medidas para predizer a quantidade de carne na carcaça. Smith \& Pearson (1986) e McLaren et al. (1987) também observaram que a AOL é um indicador da quantidade de carne presente no animal. Almeida Neto (1992) afirma ser o RP o melhor estimador para a quantidade de carne e de cortes magros na carcaça.

Os coeficientes de determinação parcial e respectiva significância do teste de hipótese para a variável RGORD (Tabela 4) evidenciam estimativas significativas para ETM, $\mathrm{P}_{1}$, AOL, RCG e RP $(8,46$; 5,$48 ; 8,17 ; 3,03$ e $8,12 \%$ ), mostrando serem estas as variáveis que mais influenciam o rendimento de gordura (RGORD). Esses resultados se assemelham aos encontrados por Oliveira (1988) quando se referem a AOL e ETM, mas diferem daqueles reportados na literatura com relação ao $\mathrm{P}_{1}$, pois a medida que aparece como a melhor para predizer a quantidade de gordura no animal é $\mathrm{P}_{2}$. RCG está de acordo com os relatos anteriores, comprovando ser uma medida razoável da qualidade da carcaça.

\section{Conclusões}

Todas as medidas de classificação estão associadas à quantidade de tecidos da carcaça, exceto o rendimento de carcaça.

É possível avaliar indiretamente os rendimentos de carne e de gordura da carcaça avaliando-se apenas as medidas de ETM, AOL, RCG e RP, sendo que, para rendimento de gordura, a avaliação de $\mathrm{P}_{1}$ também é importante.

As medidas de área de olho de lombo e espessura de toucinho, conforme propostas pelo MBCC, são as que melhor avaliam a quantidade de carne e gordura na carcaça.

\section{Literatura Citada}

ALMEIDA NETO, P.P. Parâmetros genéticos e fenotípicos de características de carcaça de suínos. Lavras: Escola Superior de Agricultura de Lavras, 1992. 71p. (Dissertação de Mestrado) - Escola Superior de Agricultura de Lavras, 1992.

ASSOCIAÇÃO BRASILEIRA DE CRIADORES DE SUÍNOS. Método brasileiro de classificação de carcaça. Rio Grande do Sul: ABCS, 1973. 17p. (Publicação Técnica, 2.)

CATALAN, G. Estimativa de parâmetros genéticos e fenotípicos em suínos Landrace, Large White e Duroc, nas fases de crescimento e terminação. Viçosa, MG: Universidade Federal de Viçosa, 1986. 129p. Dissertação (Mestrado em Zootecnia) - Universidade Federal de Viçosa, 1986.
CUTHBERTSON, A. PIDA Dissection Techniques. In: SYMPOSIUM ON METHODS OF CARCASS EVAluAtion, 1968, Dublin. Proceedings...Dublin, European Association for Animal Production, 1968. 8p.

DRAPER, N.R.; SMITH, H. Applied regression analysis. 2.ed. New York: John Wiley, 1981. 709p.

FÁVERO, J.A. Tendências da tipificação de carcaças e da qualidade da carne suína no Brasil. In: CONGRESSO BRASILEIRO DE VETERINÁRIOS ESPECIALISTAS EM SUÍNOS, 4., 1989, Itapema. Anais... Itapema: 1989. p.7-10.

IRGANG, R.; GUIDONI, A.L.; BERLITZ, D. et al. Medidas de espessura de toucinho e de profundidade de músculo para estimar rendimento de carne magra em carcaças de suínos. Revista Brasileira de Zootecnia, v.27, n.5, p.928-935, 1998.

JOHNSON, R.A.; WICHERN, D.W. Applied multivariate statistical analysis. 2.ed. New York: Prentice Hall, 1988. 607p.

JOYAL, S.M.; JONES, S.D.M.; KENNEDY, B.W. Evaluation of electronic meat-measuring equipment in predicting carcass composition in the live pig. Animal Production, v.45, n.1, p.97-102, 1987.

KEMPSTER, A.J.; CHADWICK, J.P.; JONES, D.W. et al. An evaluation of the hennessy and chong fat depth indicator, and the ulster probe, for use in pig carcass classification and grading. Animal Production, v.33, p.319-324, 1981.

McLAREN, D.C.; BUCHANAN, D.S.; JONHSON, R.K. Indivudual heterosis and breed effects for postweaning performance and carcass traits in four breed of swine. Journal of Animal Science, v.64, n.1, p.83-98, 1987.

OLIVEIRA, A.I.G. Aspectos genéticos das características físicas das carcaças de suínos em cruzamentos dialélicos. Viçosa, MG: Universidade Federal de Viçosa, 1988. 97p. Tese (Doutorado em Zootecnia) - Universidade Federal de Viçosa, 1988.

QUIJANDRIA, B.; WOODARD, J.R.; ROBISON, O.W. Genetic and environmental effects on live and carcass traits at the north Carolina swine evaluation station. Journal of Animal Science, v.31, n.1, p.652-655, 1970.

ROSTAGNO, H.S. et al. Composição de alimentos e exigências nutricionais de aves e suínos (tabelas brasileiras). Viçosa: Universidade Federal de Viçosa, 1994. 61p.

STATISTICAL ANALYSES SYSTEM - SAS. SAS/STAT. User's guide. Version 6.12. 4.ed. Cary: 1995. v.2, 1686p.

SCHINCKEL, A.P.; FORREST, J.C.; WAGNER, J.R. et al. Evaluation of B-Mode (real-time) and A-Mode ultrasoud. Swine Day, v.1, n.1, p.63-67, 1994.

SCHNEIDER, J.F.; CHRISTIAN, L.L.; KUHLERS, D.L. Crossbreeding in swine: genetic effects on pig growth and carcass merit. Journal of Animal Science, v.54, n.4, p.747-756, 1982.

SMITH, W.C.; PEARSON, G. Comparative voluntary feed intakes, growth performance, carcass compositions and meat quality of Large White, Landrace and Duroc pigs. New Zealand Journal of Experimental Agriculture, v.14, n.1, p.43-50, 1986. 